SLAC-PUB-9197

April 2002

\title{
Physics Opportunities at a Photon-Photon Collider
}

\author{
Stanley J. Brodsky \\ Stanford Linear Accelerator Center \\ 2575 Sand Hill Road, Menlo Park, CA 94025 \\ e-mail: sjbth@slac.stanford.edu
}

Invited Talk presented at the

4th International Workshop On Electron-Electron Interactions

at TeV Energies (E- E- 01)

University of California, Santa Cruz, California

7-9 December 2001

\footnotetext{
*Work by the Department of Energy, contract DE-AC03-76SF00515.
} 


\begin{abstract}
The advent of back-scattered laser beams for $e^{ \pm} e^{-}$colliders will allow detailed studies of a large array of high energy $\gamma \gamma$ and $\gamma e$ collision processes with polarized beams. These include tests of electroweak theory in photon-photon annihilation such as $\gamma \gamma \rightarrow W^{+} W^{-}, \gamma \gamma \rightarrow$ Higgs bosons, and higher-order loop processes, such as $\gamma \gamma \rightarrow \gamma \gamma, Z \gamma, H^{0} Z^{0}$ and $Z Z$. Methods for measuring the anomalous magnetic and quadrupole moments of the $W$ and $Z$ gauge bosons to high precision in polarized electron-photon and photon-photon collisions are discussed. Since each photon can be resolved into a $W^{+} W^{-}$pair, high energy photon-photon collisions can also provide a remarkably background-free laboratory for studying $W W$ collisions and annihilation. I also review high energy $\gamma \gamma$ and $e \gamma$ tests of quantum chromodynamics, including the production of two gluon jets in photon-photon collisions, deeply virtual Compton scattering on a photon target, and leading-twist single-spin asymmetries for a photon polarized normal to a production plane. Exclusive hadron production processes in photon-photon collisions provide important tests of QCD at the amplitude level, particularly as measures of hadron distribution amplitudes which are also important for the analysis of exclusive semi-leptonic and two-body hadronic $B$-decays.
\end{abstract}

\title{
1 Introduction
}

One of the most important areas of investigation at the next electron-positron linear collider will be the study of photon-photon collisions. Since photons couple directly to all fundamental fields carrying the electromagnetic current-leptons, quarks, $W^{\prime} s$, supersymmetric particles, etc.- - high energy $\gamma \gamma$ collisions will provide a comprehen-

sive laboratory for exploring virtually every aspect of the Standard Model and its extensions [1].

When a polarized laser beam Compton-scatters on a polarized electron beam, each electron is effectively converted into a polarized photon with a high fraction of its energy. The effective luminosity and energy of photon-photon collisions from back-scattered laser beams [2, 3] is expected to be comparable to that of the primary electron-positron collisions. As reported by Asner and Early [4], the technology for the required high-powered lasers is well along in development. There are proposals to test this technology using elements of the SLAC Linear Collider. The high energy luminosity, and polarization of the back-scattered laser beams collisions has the potential to make photon-photon collisions a key component of the physics program of the next linear collider [5]. Polarized electron-photon collisions are another important byproduct of this program.

Photon-photon collisions can be classified as follows: (A) The photons can annihilate into a charged pair such as $\gamma \gamma \rightarrow W^{+} W^{-}, q \bar{q}$, lepton pairs or charged Higgs; (B) 
the photons can produce neutral pairs via loop diagrams such as $\gamma \gamma \rightarrow Z^{0} Z^{0}, \gamma Z^{0}$ and $\gamma \gamma \rightarrow g g$; or $(\mathrm{C})$ the photons can each couple to separate charged pairs which scatter by a gauge particle exchange: $\gamma \gamma \rightarrow q_{1} \bar{q}_{1} q_{2} \bar{q}_{2}$; (D) the photons can fuse to produce a single even $C$ resonance such as a neutral Higgs, an $\eta_{b}$, or $\chi_{b}$ higher orbital state. Exclusive hadronic final states such as meson or baryon pairs can be formed. In each case, a state of even charge conjugation $C$ is produced in a general partial wave.

A unique advantage of a photon-photon collider is its potential to produce and determine the properties of fundamental $C=+$ resonances such as the Higgs boson [6]. One can also use the transverse polarization of the colliding photons to distinguish the parity of the resonance: the coupling for a scalar resonance is $\epsilon_{1} \cdot \epsilon_{2}$ versus $\epsilon_{1} \times k_{1} \cdot \epsilon_{2}$ for the pseudoscalar. More generally, one can use polarized photon-photon scattering to study $\mathrm{CP}$ violation in the fundamental Higgs to two-photon couplings [7, 8]. In the case of electron-photon collisions, one can use the transverse momentum fall-off of the recoil electron in $e \gamma \rightarrow e H^{0}$ to measure the fall-off of the $\gamma \rightarrow$ Higgs transition form factor and thus check the mass scale of the internal massive quark and $W$ loops coupling to the Higgs [9]. The cross sections for pairs of scalars, fermions or vectors particles are all significantly larger (by about one order of magnitude) in $\gamma \gamma$ collisions than in $e^{+} e^{-}$collisions, as demonstrated in Fig 11.

Unlike the $e^{+} e^{-}$annihilation cross section, which falls at least as fast as $1 / s$, many of the $\gamma \gamma$ cross sections increase with energy. The energy dependence of a cross section follows from the spin of the exchanged quanta. Using Regge analysis, a twobody cross section $\frac{d \sigma}{d t} \propto s^{2 \alpha_{R}(t)-2} \beta(t)$ at fixed $t$ where $\alpha_{R}$ is the spin of the exchanged particle or effective trajectory. For example, the $\gamma \gamma \rightarrow W^{+} W^{-}$differential cross section is constant at high energies since the spin of the exchanged $W$ is $\alpha_{R}=j=1$. In fact, after integration over phase space, the cross section for pairs of vector bosons in photon-photon collisions increases logarithmically with energy. This is in contrast to $\sigma\left(e^{+} e^{-} \rightarrow W^{+} W^{-}\right)$which produces a single $W^{ \pm}$pair in one partial wave and falls as $1 / s$. It is also interesting that a dominant two-jet high $p_{T}$ reaction in photonphoton collisions at high energies $s \gg p_{T}^{2}$ is $\gamma \gamma \rightarrow g g$ which proceeds via two quark loops coupling via gluon exchange in the $t$ channel 11.

\section{Standard Model Tests}

Since each photon can be resolved into a $W^{+} W^{-}$pair, high energy photon-photon collisions produce equivalent effective $W^{ \pm}$beams, thus providing a remarkably backgroundfree laboratory for studying $W W$ interactions and testing for any anomalous magnetic and quadrupole couplings. The interacting vector bosons can scatter pair-wise or annihilate; e.g., they can annihilate into a Standard Model Higgs boson or a pair of top quarks. There is thus a large array of tests of electroweak theory possible in photon-photon collisions. The splitting function for $\gamma \rightarrow W^{+} W^{-}$can be relatively flat for some $W$ helicities, so that one has a high probability for the $W$ 's to scatter 

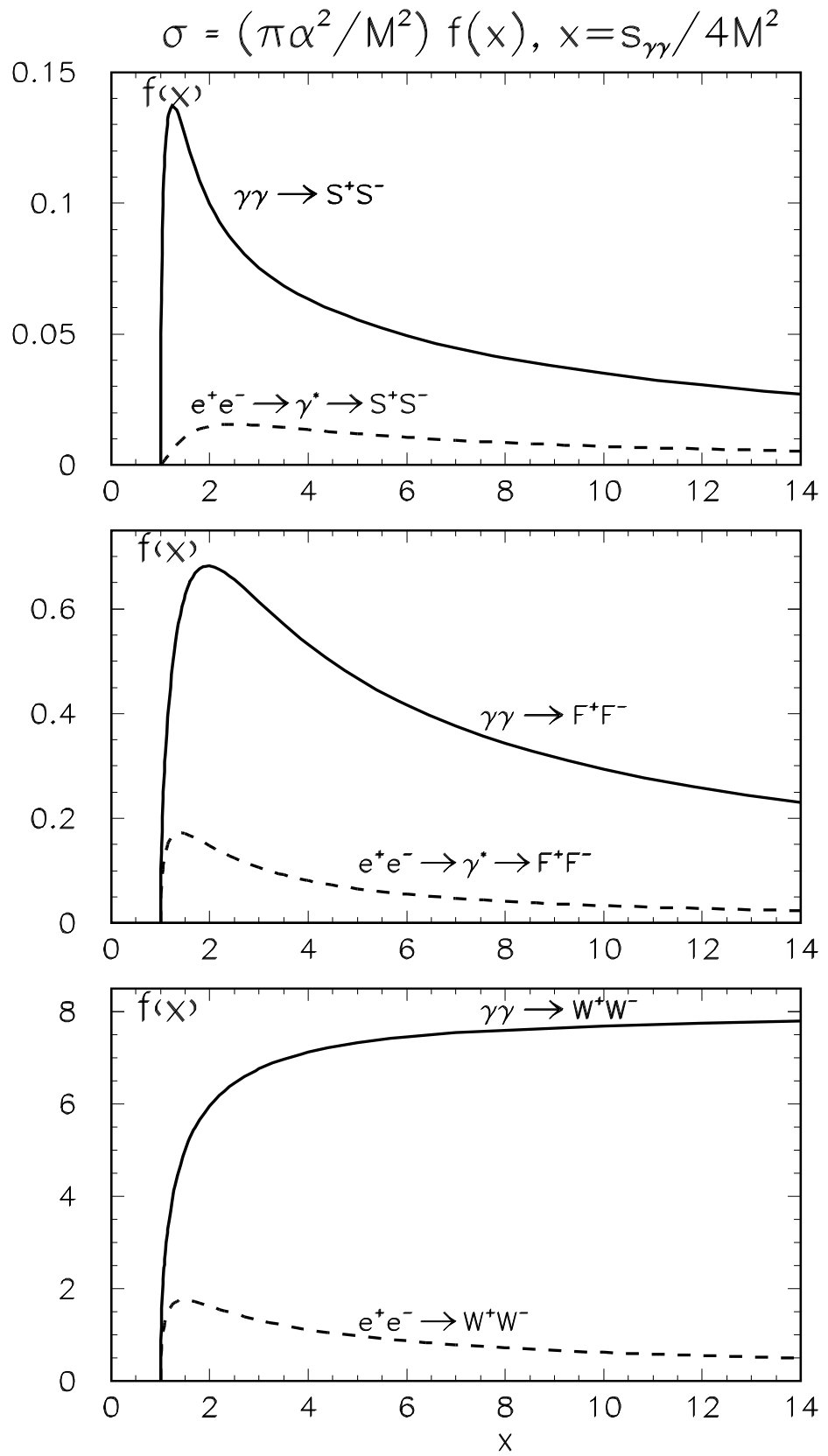

Figure 1: Comparison between cross sections for charged pair production in unpolarized $e^{+} e^{-}$and $\gamma \gamma$ collisions. S (scalars), $\mathrm{F}$ (fermions), $\mathrm{W}$ ( $W$ bosons); $\sqrt{s}$ is the invariant mass (c.m.s. energy of colliding beams). The contribution of the $Z^{0}$ boson to the production of $\mathrm{S}$ and $\mathrm{F}$ in $e^{+} e^{-}$collisions was not included. From Boos et al. [10]. 
with a high fraction of the energy of the photon. One can thus study tree graphs contributions derived from photon, Z, or Higgs exchange in the $t$-channel, and in the case of identical $W$ 's, the additional u-channel amplitudes. In the case of oppositelycharged $W^{\prime}$ 's, s-channel annihilation processes such as $W^{+} W^{-} \rightarrow t \bar{t}$ contribute. The largest cross sections will arise if the $W$ 's obey a strongly coupled theory; in this case the longitudinal $W$ 's scattering amplitude saturates unitarity and the corresponding $\gamma \gamma \rightarrow W W W W$ cross section will be maximal. The cross sections of many Standard Model processes are illustrated in Fig. 2. Reviews of this physics are given in the references. [10, 12, 13, 14, 15]

One of the most important applications of two photon physics is the direct production of $W$ pairs. By using polarized back-scattered laser beams, one can in principle study $\gamma \gamma \rightarrow W^{+} W^{-}$production as a function of initial photon helicities as well as resolve the $W$ helicities through their decays. The study of $\gamma \gamma \rightarrow W^{+} W^{-}$is complimentary to the corresponding $e^{+} e^{-} \rightarrow W^{+} W^{-}$channel, but it also can check for the presence of anomalous four-point $\gamma \gamma \rightarrow W W$ interactions not already constrained by electromagnetic gauge invariance, such as the effects due to $W^{*}$ exchange.

A main focus of the pair production measurements are the values of the $W$ magnetic moment $\mu_{W}=\frac{e}{2 m_{W}}(1-\kappa-\lambda)$ and quadrupole moment $Q_{W}=-\frac{e}{M_{W}^{2}}(\kappa-\lambda)$. The Standard Model predicts $\kappa=1$ and $\lambda=0$, up to radiative corrections analogous to the Schwinger corrections to the electron anomalous moment. The anomalous moments are thus defined as $\mu_{A}=\mu_{W}-\frac{e}{M_{W}}$ and $Q_{A}=Q_{W}+\frac{e}{M_{W}^{2}}$.

The fact that $\mu_{A}$ and $Q_{A}$ are close to zero is actually a general property of any spin-one system if its size is small compared to its Compton scale. For example, consider the Drell-Hearn-Gerasimov sum rule [16, 17] for the $W$ magnetic moment: $\mu_{A}^{2}=\left(\mu-\frac{e}{M}\right)^{2}=\frac{1}{\pi} \int_{\nu_{t h}}^{\infty} \frac{d \nu}{\nu}\left[\sigma_{P}(\nu)-\sigma_{A}(\nu)\right]$. Here $\sigma_{P(A)}$ is the total photoabsorption cross section for photons on a $W$ with (anti-) parallel helicities. As the radius of the $W$ becomes small, or its threshold energy for inelastic excitation becomes large, the DHG integral and hence $\mu_{A}^{2}$ vanishes. Hiller and I have shown [18 that this argument can be generalized to the spin-one anomalous quadrupole moment as well, by considering one of the unsubtracted dispersion relations for near-forward $\gamma$ spin-one Compton scattering [19]:

$$
\begin{aligned}
& \mu_{A}^{2}+\frac{2 t}{M_{W}^{2}}\left(\mu_{A}+\frac{M_{2}}{W} Q_{A}\right)^{2}= \\
& \frac{1}{4 \pi} \int_{\nu_{t h}}^{\infty} \frac{d \nu^{2}}{(\nu-t / 4)^{3}} \operatorname{Im}\left(f_{P}(s, t)-f_{A}(s, t)\right) .
\end{aligned}
$$

Here $\nu=(s-u) / 4$. One again sees that in the point-like or high threshold energy limit, both $\mu_{A} \rightarrow 0$, and $Q_{A} \rightarrow 0$. This result applies to any spin-one system, even to the deuteron or the $\rho$. The essential assumption is the existence of the unsubtracted dispersion relations; i.e., that the anomalous moments are in principle computable quantities. 


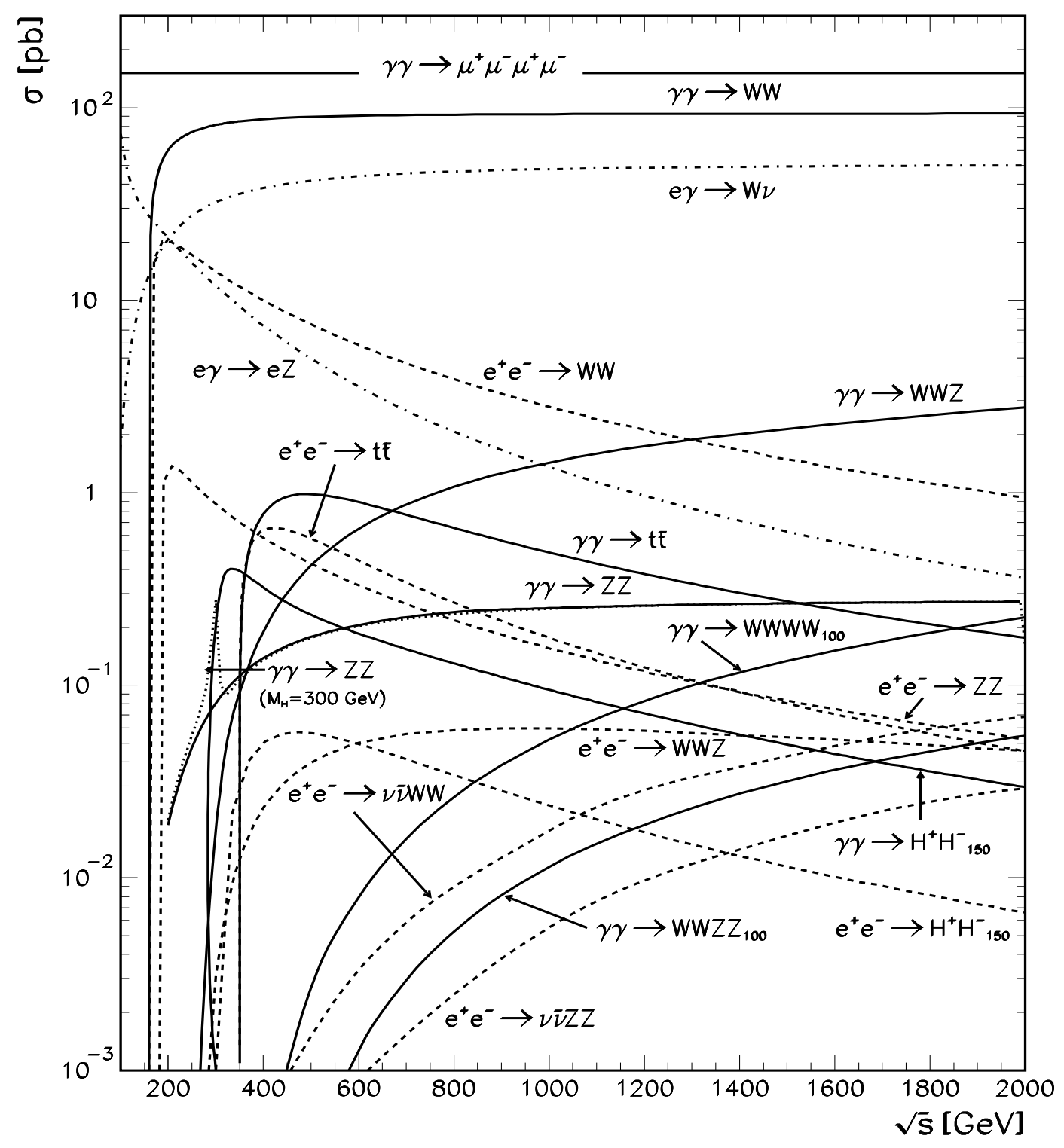

Figure 2: Typical (unpolarized) cross sections in $\gamma \gamma, \gamma e$ and $e^{+} e^{-}$collisions. Solid, dash-dotted and dashed curves correspond to $\gamma \gamma$, $\gamma e$ and $e^{+} e^{-}$modes respectively. Unless indicated otherwise the neutral Higgs mass was taken to be $100 \mathrm{GeV}$. For charged Higgs pair production, $M_{H^{ \pm}}=150 \mathrm{GeV}$ was assumed. From Boos et al. [10]. 
In the case of the $W$, the finite size correction is expected to be order $m^{2} / \Lambda^{2}$, since the underlying composite theory should be chiral to keep the $W$ mass finite as the composite scale $\Lambda$ becomes large [20]. Thus the fact that a spin-one system has nearly canonical values for its moments signals that it has a small internal size; however, it does not necessarily imply that it is a gauge field.

Yehudai [21] has made extensive studies of the effect of anomalous moments on different helicity amplitude contributing to $\gamma \gamma \rightarrow W^{+} W^{-}$cross section. The empirical sensitivity to anomalous couplings from $\gamma \gamma$ reactions is comparable and complimentary to that of $e^{+} e^{-} \rightarrow W^{+} W^{-}$. A comprehensive analysis of fermion processes in photon-photon collisions is given by Layssac and Renard [22].

As emphasized by Jikia and Tkabladze [23], pairs of neutral gauge bosons can be produced in $\gamma \gamma$ reactions through one loop amplitudes in the Standard Model at a rate which should be accessible to the NLC. Leptons, quarks, and $W$ all contribute to the box graphs. The fermion and spin-one exchange contributions to the $\gamma \gamma \rightarrow \gamma \gamma$ scattering amplitude have the characteristic behavior $\mathcal{M} \sim s^{0} f(t)$ and $\mathcal{M} \sim i s f(t)$ respectively. The latter is the dominant contribution at high energies, so one can use the optical theorem to relate the forward imaginary part of the scattering amplitude to the total $\gamma \gamma \rightarrow W^{+} W^{-}$cross section. The resulting cross section $\sigma(\gamma \gamma \rightarrow \gamma \gamma)$ is of order $20 \mathrm{fb}$ at $\sqrt{s}_{\gamma \gamma}$, corresponding to 200 events/year at an NLC with luminosity $10 \mathrm{fb}^{-1}$ [10]. The corresponding $\gamma \gamma \rightarrow H^{0} Z^{0}$ process has been analyzed by Gounaris, Porfyriadis and Renard [24].

A single top quark can be produced in electron-photon collisions at an NLC through the process $e^{-} \gamma \rightarrow W^{-} t \nu$ [25]. See Fig. 3. [26] This process can be identified through the $t \rightarrow W^{+} b$ decay with $W \rightarrow \ell \bar{\nu}$. The rate is strongly polarization dependent and is sensitive to the structure of the $V_{t b}$ matrix element, possible fourth generation quarks, and anomalous couplings. An interesting background is the virtual $W$ process $e \gamma \rightarrow W^{*}-\nu \rightarrow W^{-} H \nu$, where the Higgs boson decays to $b \bar{b}$ and $W^{-} \rightarrow \ell \bar{\nu}$.

Schmidt, Rizzo, and I [27, 28] have shown that one can use the sign change of the integrand of the DHG sum rule to test the canonical couplings of the Standard Model and to isolate the higher order radiative corrections. For example, consider the reactions $\gamma \gamma \rightarrow q \bar{q}, \gamma e \rightarrow W \nu$ and $\gamma e \rightarrow Z e$ which can be studied with back-scattered laser beams. In contrast to the time-like process $e^{+} e^{-} \rightarrow W^{+} W^{-}$, the $\gamma \gamma$ and $\gamma e$ reactions are sensitive to the anomalous moments of the gauge bosons at $q^{2}=0$. The vanishing of the logarithmic integral of $\Delta \sigma$ in the Born approximation implies that there must be a center-of-mass energy, $\sqrt{s}_{0}$, where the polarization asymmetry $A=\Delta \sigma / \sigma$ possesses a zero, i.e., where $\Delta \sigma(\gamma e \rightarrow W \nu)$ reverses sign. The cancellation of the positive and negative contributions [29] of $\Delta \sigma(\gamma e \rightarrow W \nu)$ to the DHG integral is evident in Fig. 团. We find strong sensitivity of the position of this zero or "crossing point" (which occurs at $\sqrt{s}_{\gamma e}=3.1583 \ldots M_{W} \simeq 254 \mathrm{GeV}$ in the SM) to modifications of the SM trilinear $\gamma W W$ coupling and thus can lead to high precision constraints. In addition to the fact that only a limited range of energy is required, the polarization 


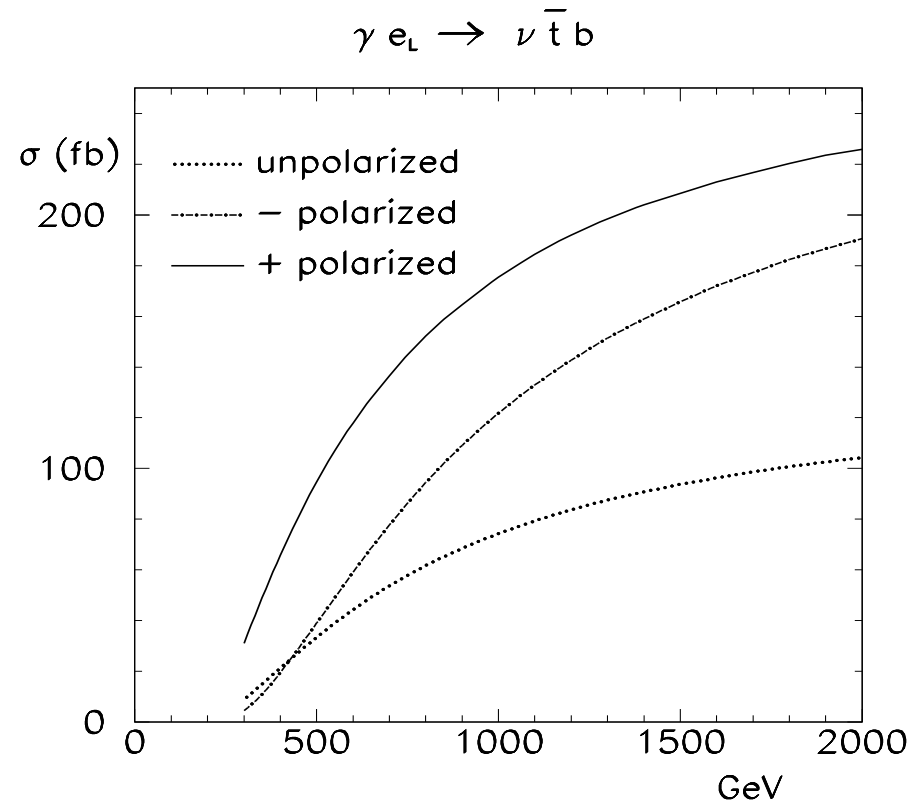

Figure 3: Single top quark production cross section in $\gamma e$ collisions. From Boos et al. 26.

asymmetry measurements have the advantage that many of the systematic errors cancel in taking cross section ratios. This technique can clearly be generalized to other higher order tree-graph processes in the Standard Model and supersymmetric gauge theory. The position of the zero in the photoabsorption asymmetry thus provides an additional weapon in the arsenal used to probe anomalous trilinear gauge couplings.

\section{Inclusive QCD Tests}

Because of the simplicity of its initial state, two-photon collisions provide an important laboratory for testing coherent and incoherent effects in quantum chromodynamics. In QCD events where each photon is resolved [30, 31] in terms of its intermediate quark and gluon states, $\gamma \gamma$ collisions resemble point-like meson-meson collisions. One can study detailed features of $\gamma \gamma \rightarrow t \bar{t}$ at threshold and its final state evolution. In the case of single or double diffractive two-photon events, one can study fundamental aspects of pomeron and odderon $t$-channel physics. For example, the energy asymmetry of charm quark versus anti-charm jets in four-jet reactions can measure the interference of the pomeron and odderon 32].

Consider, the cross section for producing two quark pairs. $\gamma \gamma \rightarrow q_{1} \bar{q}_{1} q_{2} \bar{q}_{2}$, which 


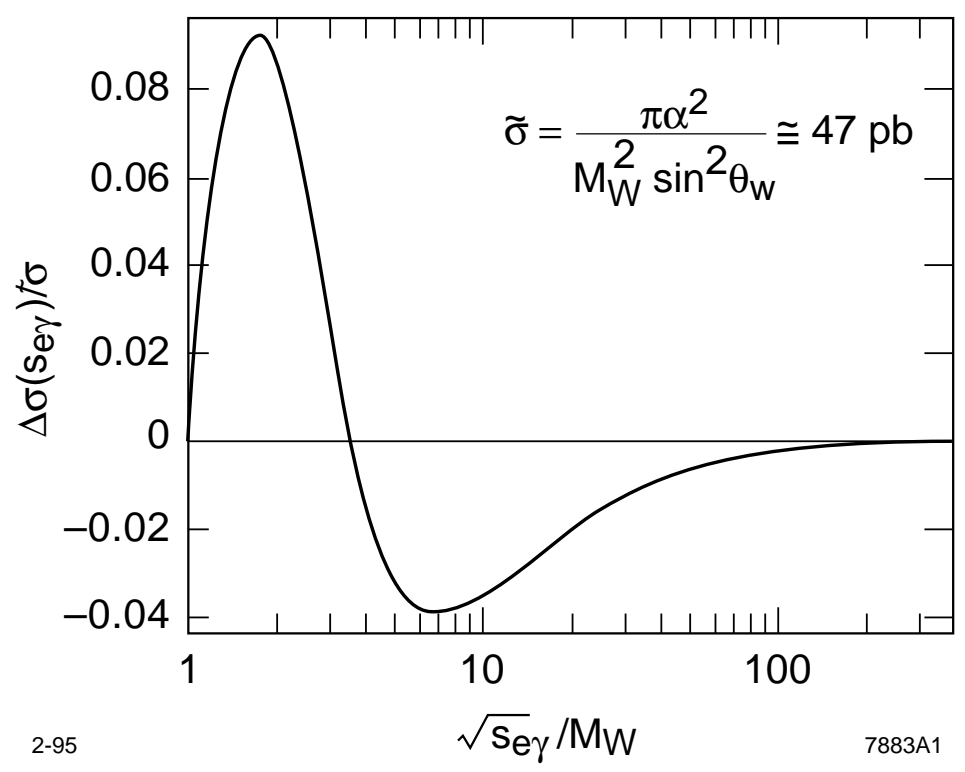

Figure 4: The Born cross section difference $\Delta \sigma$ for the Standard Model process $\gamma e \rightarrow W \nu$ for parallel minus antiparallel electron/photon helicities as a function of $\log \sqrt{s}_{e \gamma} / M_{W}$ The logarithmic integral of $\Delta \sigma$ vanishes in the classical limit. 27]

can be described as the interaction of two color dipoles [33, 34]. If one of the heavy quark pairs is a charm or bottom quark pair with a small color dipole moment, then the multiple gluonic exchange graphs will Reggeize into a hard pomeron, and one predict a strong energy growth of the production cross section similar to that observed at HERA in diffractive charm electroproduction [35]. The QCD description of the hard QCD pomeron is given by the BFKL analysis [36. There has recently been progress in stabilizing the BFKL predictions at next-to-leading-order by using BLM scale fixing. [37]

\section{The Photon Structure Functions}

One can also utilize electron-photon collisions at a linear collider to test the shape and growth of the photon structure functions 33, 39, 40, 31 The back-scattered laser beam provides a high energy polarized target photon, and the neutral current probe is obtained by tagging the scattered electron at momentum transfer squared $Q^{2}$. One can also reconstruct the charged current contributions where the electron scatters into a neutrino from calorimetric measurements of the recoiling system. It also should be possible to identify the separate charm, bottom, top and $W$ contributions to the photon structure functions.

The photon structure functions receive hadron-like contributions from the pho- 
ton's resolved Fock components as well as its direct component derived from the $\gamma^{*} \gamma \rightarrow q \bar{q}$ time-like QCD Compton amplitude. Because of the direct contributions, the photon structure functions obey an inhomogeneous evolution equation. The result, as first shown by Witten [41] is that the leading order QCD structure functions of the photon have a unique scaling behavior: $F_{1}\left(x, Q^{2}\right)=h(x) \ln \frac{Q^{2}}{\Lambda^{2}}, F_{2}\left(x, Q^{2}\right)=f_{2}(x)$, $F_{3}\left(x, Q^{2}\right)=f_{3}^{\text {Box }}(x)$.

The most characteristic behavior of the photon structure function $F_{2}^{\gamma}\left(x, Q^{2}\right)$ in QCD is its continuous linear rise of with $\log Q^{2}$ at fixed $x$. As emphasized by Peterson, Walsh and Zerwas [42, the fact that this tree graph behavior is preserved to all orders in perturbation theory is due to the balance in QCD between the increase of the phase space for gluon emission in the scattering processes versus the decreasing strength of the gluon coupling due to asymptotic freedom. Although the logarithmic rise of the Born approximation result is preserved, the shape of $h(x)$ is modified by the QCD radiation. If the running coupling constant were to freeze to a constant value at large momentum transfer, the photon structure function stops rising at high $Q^{2}$ due to the increased phase space for gluon radiation. Thus probing the QCD photon structure functions at the high momentum transfers available at the NLC will provide a valuable test of asymptotic freedom.

\section{The Photon Structure Function and Final-State Interactions}

Recently, Hoyer, Marchal, Peigne, and Sannino and I 43] have challenged the common view that structure functions measured in deep inelastic lepton scattering are determined by the probability of finding quarks and gluons in the target hadron. We show that this is not correct in gauge theory. Gluon exchange between the fast, outgoing partons and target spectators, which is usually assumed to be an irrelevant gauge artifact, affects the leading twist structure functions in a profound way. This observation removes the apparent contradiction between the projectile (eikonal) and target (parton model) views of diffractive and small $x_{\text {Bjorken }}$ phenomena. The diffractive scattering of the fast outgoing quarks on spectators in the target in turn causes shadowing in the DIS cross section. Thus the depletion of the nuclear structure functions is not intrinsic to the wave function of the nucleus, but is a coherent effect arising from the destructive interference of diffractive channels induced by final-state interactions. This is consistent with the Glauber-Gribov interpretation of shadowing as a rescattering effect. Similar effects can be present in the photon structure function; i.e., the photon structure function is modified by rescattering of the struck quark with the photon's spectator system. 


\section{Single-Spin Asymmetries in Photon-Photon Col- lisions}

Measurements from the HERMES and SMC collaborations show a remarkably large azimuthal single-spin asymmetries $A_{U L}$ and $A_{U T}$ of the proton in semi-inclusive pion leptoproduction $\gamma^{*}(q) p \rightarrow \pi X$. Recently, Dae Sung Hwang and Ivan Schmidt and I [44] have shown that final-state interactions from gluon exchange between the outgoing quark and the target spectator system lead to single-spin asymmetries in deep inelastic lepton-proton scattering at leading twist in perturbative QCD; i.e., the rescattering corrections are not power-law suppressed at large photon virtuality $Q^{2}$ at fixed $x_{b j}$. The existence of such single-spin asymmetries requires a phase differ-

ence between two amplitudes coupling the proton target with $J_{p}^{z}= \pm \frac{1}{2}$ to the same final-state, the same amplitudes which are necessary to produce a nonzero proton anomalous magnetic moment. We have shown that the exchange of gauge particles between the outgoing quark and the proton spectators produces a Coulomb-like complex phase which depends on the angular momentum $L^{z}$ of the proton's constituents and is thus distinct for different proton spin amplitudes. The single-spin asymmetry which arises from such final-state interactions does not factorize into a product of distribution function and fragmentation function, and it is not related to the transversity distribution $\delta q(x, Q)$ which correlates transversely polarized quarks with the spin of the transversely polarized target nucleon. These effects highlight the unexpected importance of final and initial state interactions in QCD observables.

Final state interactions will also lead to new types of single spin asymmetries in photon-photon collisions. For example, in $\gamma^{*} \gamma \rightarrow \pi X$ and $\gamma^{*} \gamma \rightarrow$ jet $X$ we expect $T$ odd correlations of the type $\vec{S}_{\gamma} \cdot \vec{q} \times \vec{p}$ where $\vec{S}_{\gamma}$ is the polarization of the real photon, $\vec{q}$ is the beam direction of an incident virtual photon, and $\vec{p}$ is the direction of a produced quark or hadron. The resulting asymmetry of the photon polarized normal to the production plane will be leading twist. As in the proton target case, the single-spin asymmetry will be sensitive to orbital angular momentum in the photon wavefunction and details of the photon structure at the amplitude level.

\section{Single and Double Diffraction in Photon-Photon Collisions}

The high energies of a photon-photon collider will make the study of double diffractive $\gamma \gamma \rightarrow V^{0} V^{0}$ and semi-inclusive single diffractive processes $\gamma \gamma \rightarrow V^{0} X$ in the Regge regime $s>|t|$ interesting. Here $V^{0}=\rho, \omega \phi, J / \psi, \cdots$ If $|t|$ is taken larger than the QCD confinement scale, then one has the potential for a detailed study of fundamental Pomeron processes and its gluonic composition. As in the case of large angle exclusive $\gamma \gamma$ processes, the scattering amplitude is computed by convoluting the hard scattering PQCD amplitude for $\gamma \gamma \rightarrow q \bar{q} q \bar{q}$ with the vector meson distribution amplitudes. 
The two gluon exchange contribution dominates in the Regge regime [45], giving a characteristic exclusive process scaling law of order $\frac{d \sigma}{d t}\left(\gamma \gamma \rightarrow V^{0} V^{0}\right) \sim \alpha_{s}^{4}(t) / t^{6}$. Ginzburg, Ivanov and Serbo [46] have emphasized that the corresponding $\gamma \gamma \rightarrow$ pseudoscalar and tensor meson channels can be used to isolate the Odderon exchange contribution, contributions related at a fundamental level to three gluon exchange.

In addition, the photon can diffractively dissociate into quark pairs $\gamma e \rightarrow q \bar{q} e^{\prime}$ by Coulomb scattering on the incoming electron. This measures the transverse derivative

of the photon wavefunction $\frac{\partial}{\partial k_{\perp}} \psi_{q \bar{q}}\left(x, k_{\perp}, \lambda_{i}\right)$. This is the analog of the E791 experiment at Fermilab [47 which resolved the pion light-front wavefunction by diffractive dissociation $\pi A \rightarrow q \bar{q} A^{\prime}$ on a nuclear target. The results of the diffractive pion experiment are consistent with color transparency, and the momentum partition of the jets conforms closely with the shape of the asymptotic distribution amplitude, $\phi_{\pi}^{\text {asympt }}(x)=\sqrt{3} f_{\pi} x(1-x)$, corresponding to the leading anomalous dimension solution [48] to the perturbative QCD evolution equation.

\section{Other QCD Tests in Photon-Photon Collisions}

Two-photon annihilation $\gamma^{*}\left(q_{1}\right) \gamma^{*}\left(q_{2}\right) \rightarrow$ hadrons for real and virtual photons can thus provide some of the most detailed and incisive tests of QCD. Among the processes of special interest are:

1. the production of four jets such as $\gamma \gamma \rightarrow c \bar{c} c \bar{c}$ can test Fermi-color statistics for charm quarks by checking for the interference effects of like sign quarks [49].

2. the total two-photon annihilation hadronic cross section $\sigma\left(s, q_{1}^{2}, q_{2}^{2}\right)$, which is related to the light-by-light hadronic contribution to the muon anomalous moment;

3. the formation of $C=+$ hadronic resonances, which can reveal exotic states such as $q \bar{q} g$ hybrids and discriminate gluonium formation [50, 51]. The production of the $\eta_{B}$ and $\chi_{B}$ states are essentially unexplored in QCD. [52]

4. single-hadron processes such as $\gamma^{*} \gamma^{*} \rightarrow \pi^{0}$, which test the transition from the anomaly-dominated pion decay constant to the short-distance structure of currents dictated by the operator-product expansion and perturbative QCD factorization theorems;

5. hadron pair production processes such as $\gamma^{*} \gamma \rightarrow \pi^{+} \pi^{-}, K^{+} K^{-}, p \bar{p}$, which at fixed invariant pair mass measures the $s \rightarrow t$ crossing of the virtual Compton amplitude [53, 48]. I discuss this further in the next section. When one photon is highly virtual, these exclusive hadron production channels are dual to the photon structure function $F_{2}^{\gamma}\left(x, Q^{2}\right)$ in the endpoint $x \rightarrow 1$ region at fixed invariant pair mass. The leading twist-amplitude for $\gamma^{*} \gamma \rightarrow \pi^{+} \pi^{-}$is sensitive to 
the $1 / x-1 /(1-x)$ moment of the $q \bar{q}$ distribution amplitude $\Phi_{\pi^{+} \pi^{-}}\left(x, Q^{2}\right)$ of the two-pion system [54, 55], the time-like extension of skewed parton distributions. In addition one can measure the pion charge asymmetry in $e^{+} e^{-} \rightarrow \pi^{+} \pi^{-} e^{+} e^{-}$ arising from the interference of the $\gamma \gamma \rightarrow \pi^{+} \pi^{-}$Compton amplitude with the time-like pion form factor [56]. At the unphysical point $s=q_{1}^{2}=q_{2}^{2}=0$, the amplitude is fixed by the low energy theorem to the hadron charge squared. The ratio of the measured $\gamma \gamma \rightarrow \Lambda \bar{\Lambda}$ and $\gamma \gamma \rightarrow p \bar{p}$ cross sections is anomalous at threshold, a fact which may be associated with the soliton structure of baryons in QCD [57, 58];

6. Exclusive or semi-inclusive channels can also be studied by coalescence of the produced quarks. An interesting example is higher generation final state such as $\gamma \gamma \rightarrow B_{c} \bar{B}_{c}$, which can have very complex angular structure [59].

7. As pointed out by Hwang [60, one can study deeply virtual Compton scattering on a photon target in $e \gamma$ collisions to determine the light-cone wavefunctions and other features of the photon [61.

\section{The Photon-to-Pion Transition Form Factor and the Pion Distribution Amplitude}

The simplest and perhaps most elegant illustration of an exclusive reaction in QCD is the evaluation of the photon-to-pion transition form factor $F_{\gamma \rightarrow \pi}\left(Q^{2}\right)$ which is measurable in single-tagged two-photon $e e \rightarrow e e \pi^{0}$ reactions. The form factor is defined via the invariant amplitude $\Gamma^{\mu}=-i e^{2} F_{\pi \gamma}\left(Q^{2}\right) \varepsilon^{\mu \nu \rho \sigma} p_{\nu}^{\pi} \varepsilon_{\rho} q_{\sigma}$. As in inclusive reactions, one must specify a factorization scheme which divides the integration regions of the loop integrals into hard and soft momenta, compared to the resolution scale $\widetilde{Q}$. At leading twist, the transition form factor then factorizes as a convolution of the $\gamma^{*} \gamma \rightarrow q \bar{q}$ amplitude (where the quarks are collinear with the final state pion) with the valence light-cone wavefunction of the pion 48:

$$
F_{\gamma M}\left(Q^{2}\right)=\frac{4}{\sqrt{3}} \int_{0}^{1} d x \phi_{M}(x, \widetilde{Q}) T_{\gamma \rightarrow M}^{H}\left(x, Q^{2}\right) .
$$

The hard scattering amplitude for $\gamma \gamma^{*} \rightarrow q \bar{q}$ is $T_{\gamma M}^{H}\left(x, Q^{2}\right)=\left[(1-x) Q^{2}\right]^{-1}\left(1+\mathcal{O}\left(\alpha_{s}\right)\right)$. For the asymptotic distribution amplitude $\phi_{\pi}^{\text {asympt }}(x)=\sqrt{3} f_{\pi} x(1-x)$ one predicts 62

$$
Q^{2} F_{\gamma \pi}\left(Q^{2}\right)=2 f_{\pi}\left(1-\frac{5}{3} \frac{\alpha_{V}\left(Q^{*}\right)}{\pi}\right)
$$

where $Q^{*}=e^{-3 / 2} Q$ is the estimated BLM scale for the pion form factor in the $V$ scheme. 


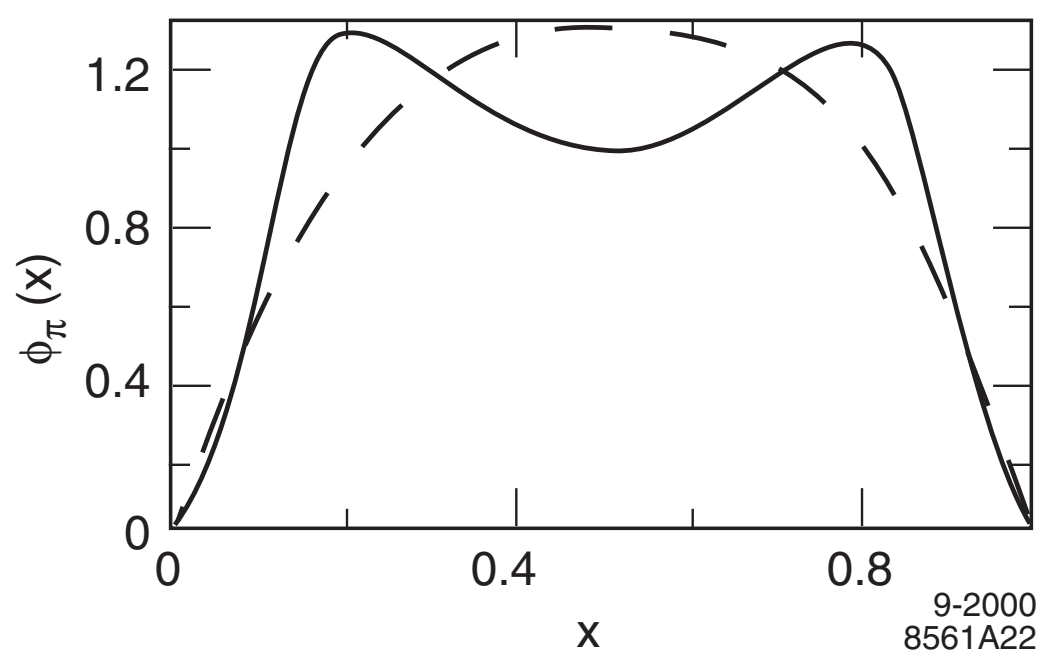

(a)

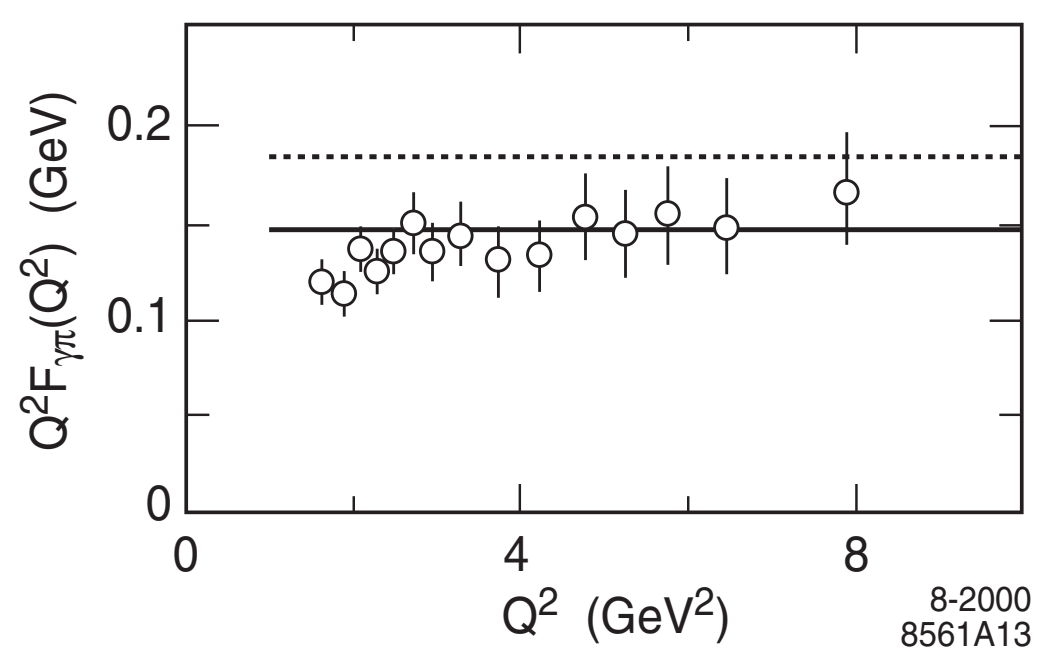

(b)

Figure 5: (a) Transverse lattice results for the pion distribution amplitude at $Q^{2} \sim 10 \mathrm{GeV}^{2}$. The solid curve is the theoretical prediction from the combined DLCQ/transverse lattice method [64]; the chain line is the experimental result obtained from di-jet diffractive dissociation [47, 65]. Both are normalized to the same area for comparison. (b) Scaling of the transition photon to pion transition form factor $Q^{2} F_{\gamma \pi^{0}}\left(Q^{2}\right)$. The dotted and solid theoretical curves are the perturbative QCD prediction at leading and next-to-leading order, respectively, assuming the asymptotic pion distribution The data are from the CLEO collaboration [63]. 
The PQCD predictions have been tested in measurements of $e \gamma \rightarrow e \pi^{0}$ by the CLEO collaboration [63] (see Fig. 5 (b)). The flat scaling of the $Q^{2} F_{\gamma \pi}\left(Q^{2}\right)$ data from $Q^{2}=2$ to $Q^{2}=8 \mathrm{GeV}^{2}$ provides an important confirmation of the applicability of leading twist QCD to this process. The magnitude of $Q^{2} F_{\gamma \pi}\left(Q^{2}\right)$ is remarkably consistent with the predicted form, assuming the asymptotic distribution amplitude and including the LO QCD radiative correction with $\alpha_{V}\left(e^{-3 / 2} Q\right) / \pi \simeq 0.12$. One could allow for some broadening of the distribution amplitude with a corresponding increase in the value of $\alpha_{V}$ at small scales. Radyushkin 66], Ong [67 and Kroll 68] have also noted that the scaling and normalization of the photon to pion transition form factor tends to favor the asymptotic form for the pion distribution amplitude and rules out broader distributions such as the two-humped form suggested by QCD sum rules [69. More detailed studies are given in the references. [70, 71, 72]

When both photons are virtual, the denominator of $T_{H}$ for the $\gamma \gamma^{*} \rightarrow \pi^{0}$ reaction becomes $(1-x) Q_{1}^{2}+x Q_{2}^{2}$ [48, 67], and the amplitude becomes nearly insensitive to the shape of the distribution amplitude once it is normalized to the pion decay constant. Thus the ratio of singly virtual to doubly virtual pion production is particularly sensitive to the shape of $\phi_{\pi}\left(x, Q^{2}\right)$ since higher order corrections and normalization errors tend to cancel in the ratio.

\section{Exclusive Two-Photon Annihilation into Hadron Pairs}

At large momentum transfer, the angular distribution of hadron pairs produced by photon-photon annihilation are among the best determinants of the shape of the meson and baryon distribution amplitudes $\phi_{M}(x, Q)$ and $\phi_{B}\left(x_{i}, Q\right)$, which control almost all exclusive processes involving a hard scale $Q$. The determination of the shape and normalization of the distribution amplitudes, which are gauge-invariant and process-independent measures of the valence wavefunctions of the hadrons, has become particularly important in view of their importance in the analysis of exclusive semi-leptonic and two-body hadronic $B$-decays [73, 74, 75, 76, 77. There has also been considerable progress both in calculating hadron wavefunctions from first principles in QCD and in measuring them using diffractive di-jet dissociation.

Much of this important two-photon physics is also accessible at low energy, high luminosity $e^{+} e^{-}$colliders, particularly for measurements of channels important in the light-by-light contribution to the muon $g-2$ and the exploration of the transition between threshold amplitudes which are controlled by low-energy effective theories such as the chiral Hamiltonian through the transition to the domain where leading-twist perturbative QCD becomes applicable. There have been almost no measurements of double-tagged events needed to unravel the separate $q_{1}^{2}$ and $q_{2}^{2}$ dependence of photon-

photon annihilation. Hadron pair production from two-photon annihilation plays a 
crucial role in unravelling the perturbative and non-perturbative structure of QCD, first by testing the validity and empirical applicability of leading-twist factorization theorems, second by verifying the structure of the underlying perturbative QCD subprocesses, and third, through measurements of angular distributions and ratios which are sensitive to the shape of the distribution amplitudes. In effect, photon-photon collisions provide a microscope for testing fundamental scaling laws of PQCD and for measuring distribution amplitudes.

Two-photon reactions, $\gamma \gamma \rightarrow H \bar{H}$ at large $s=\left(k_{1}+k_{2}\right)^{2}$ and fixed $\theta_{\mathrm{cm}}$, provide a particularly important laboratory for testing QCD since these cross-channel "Compton" processes are the simplest calculable large-angle exclusive hadronic scattering reactions. The helicity structure, and often even the absolute normalization can be rigorously computed for each two-photon channel [53]. In the case of meson pairs, dimensional counting predicts that for large $s, s^{4} d \sigma / d t(\gamma \gamma \rightarrow M \bar{M})$ scales at fixed $t / s$ or $\theta_{\mathrm{cm}}$ up to factors of $\ln s / \Lambda^{2}$. The angular dependence of the $\gamma \gamma \rightarrow H \bar{H}$ amplitudes can be used to determine the shape of the process-independent distribution amplitudes, $\phi_{H}(x, Q)$. An important feature of the $\gamma \gamma \rightarrow M \bar{M}$ amplitude for meson pairs is that the contributions of Landshoff pitch singularities are power-law suppressed at the Born level - even before taking into account Sudakov form factor suppression. There are also no anomalous contributions from the $x \rightarrow 1$ endpoint integration region. Thus, as in the calculation of the meson form factors, each fixedangle helicity amplitude can be written to leading order in $1 / Q$ in the factorized form $\left[Q^{2}=p_{T}^{2}=t u / s ; \widetilde{Q}_{x}=\min (x Q,(l-x) Q)\right]:$

$$
\begin{aligned}
\mathcal{M}_{\gamma \gamma \rightarrow M \bar{M}} & =\int_{0}^{1} d x \int_{0}^{1} d y \\
& \phi_{\bar{M}}\left(y, \widetilde{Q}_{y}\right) T_{H}\left(x, y, s, \theta_{\mathrm{cm}} \phi_{M}\left(x, \widetilde{Q}_{x}\right),\right.
\end{aligned}
$$

where $T_{H}$ is the hard-scattering amplitude $\gamma \gamma \rightarrow(q \bar{q})(q \bar{q})$ for the production of the valence quarks collinear with each meson, and $\phi_{M}(x, \widetilde{Q})$ is the amplitude for finding the valence $q$ and $\bar{q}$ with light-cone fractions of the meson's momentum, integrated over transverse momenta $k_{\perp}<\widetilde{Q}$. The contribution of non-valence Fock states are power-law suppressed. Furthermore, the helicity-selection rules [78] of perturbative QCD predict that vector mesons are produced with opposite helicities to leading order in $1 / Q$ and all orders in $\alpha_{s}$. The dependence in $x$ and $y$ of several terms in $T_{\lambda, \lambda^{\prime}}$ is quite similar to that appearing in the meson's electromagnetic form factor. Thus much of the dependence on $\phi_{M}(x, Q)$ can be eliminated by expressing it in terms of the meson form factor. In fact, the ratio of the $\gamma \gamma \rightarrow \pi^{+} \pi^{-}$and $e^{+} e^{-} \rightarrow \mu^{+} \mu^{-}$ amplitudes at large $s$ and fixed $\theta_{C M}$ is nearly insensitive to the running coupling and the shape of the pion distribution amplitude:

$$
\frac{\frac{d \sigma}{d t}\left(\gamma \gamma \rightarrow \pi^{+} \pi^{-}\right)}{\frac{d \sigma}{d t}\left(\gamma \gamma \rightarrow \mu^{+} \mu^{-}\right)} \sim \frac{4\left|F_{\pi}(s)\right|^{2}}{1-\cos ^{2} \theta_{\mathrm{cm}}} .
$$

The comparison of the PQCD prediction for the sum of $\pi^{+} \pi^{-}$plus $K^{+} K^{-}$channels 

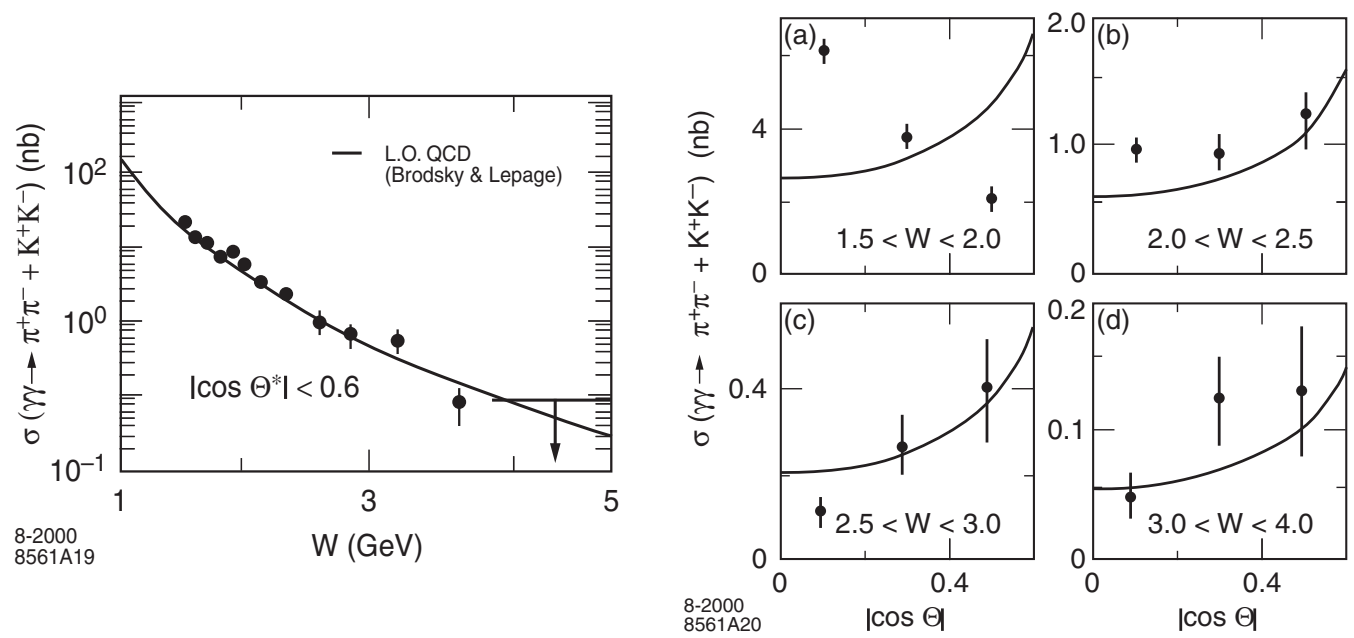

Figure 6: Comparison of the sum of $\gamma \gamma \rightarrow \pi^{+} \pi^{-}$and $\gamma \gamma \rightarrow K^{+} K^{-}$meson pair production cross sections with the scaling and angular distribution of the perturbative QCD prediction [53]. The data are from the CLEO collaboration [79].

with CLEO data 799 is shown in Fig. 6. The CLEO data for charged pion and kaon pairs show a clear transition to the scaling and angular distribution predicted by PQCD [53] for $W=\sqrt{(} s_{\gamma \gamma}>2 \mathrm{GeV}$. It is clearly important to measure the magnitude and angular dependence of the two-photon production of neutral pions and $\rho^{+} \rho^{-}$in view of the strong sensitivity of these channels to the shape of meson distribution amplitudes (see Figs. 7 and 8 ). QCD also predicts that the production cross section for charged $\rho$-pairs (with any helicity) is much larger than for that of neutral $\rho$ pairs, particularly at large $\theta_{\mathrm{cm}}$ angles. Similar predictions are possible for other helicity-zero mesons. For an alternative model based on the QCD "handbag" diagram, see the references. 80

As noted above, the analysis of exclusive $B$ decays has much in common with the analysis of exclusive two-photon reactions [81]. For example, consider the three representative contributions to the decay of a $B$ meson to meson pairs illustrated in Fig. 9. In Fig. 9(a) the weak interaction effective operator $\mathcal{O}$ produces a $q \bar{q}$ in a color octet state. A gluon with virtuality $Q^{2}=\mathcal{O}\left(M_{B}^{2}\right)$ is needed to equilibrate the large momentum fraction carried by the $b$ quark in the $\bar{B}$ wavefunction. The amplitude then factors into a hard QCD/electroweak subprocess amplitude for quarks which are collinear with their respective hadrons: $T_{H}\left([b(x) \bar{u}(1-x)] \rightarrow[q(y) \bar{u}(1-y)]_{1}[q(z) \bar{q}(1-\right.$ $z)]_{2}$ ) convoluted with the distribution amplitudes $\phi(x, Q)$ [4] of the incident and final hadrons:

$$
\begin{aligned}
& \mathcal{M}_{\text {octet }}\left(B \rightarrow M_{1} M_{2}\right)=\int_{0}^{1} d z \int_{0}^{1} d y \int_{0}^{1} d x \\
& \phi_{B}(x, Q) T_{H}(x, y, z) \phi_{M_{1}}(y, Q) \phi_{M_{2}}(z, Q) .
\end{aligned}
$$




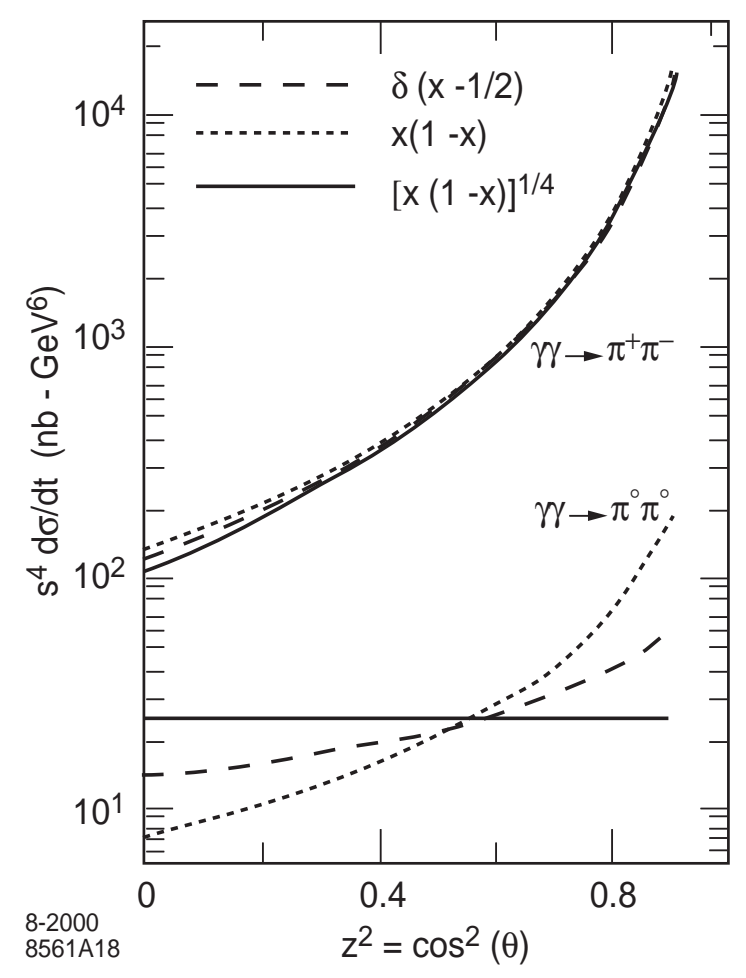

Figure 7: Predictions for the angular distribution of the $\gamma \gamma \rightarrow \pi^{+} \pi^{-}$and $\gamma \gamma \rightarrow \pi^{0} \pi^{0}$ pair production cross sections for three different pion distribution amplitudes [53].

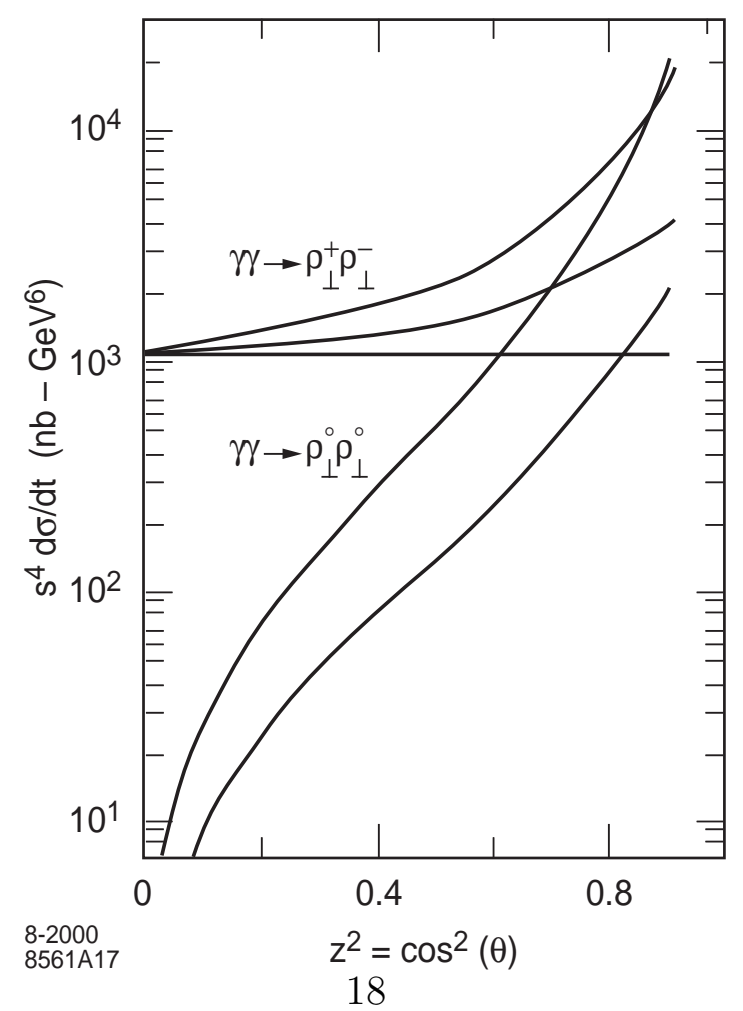

Figure 8: Predictions for the angular distribution of the $\gamma \gamma \rightarrow \rho^{+} \rho^{-}$and $\gamma \gamma \rightarrow \rho^{0} \rho^{0}$ pair production cross sections for three different $\rho$ distribution amplitudes as in Fig. 


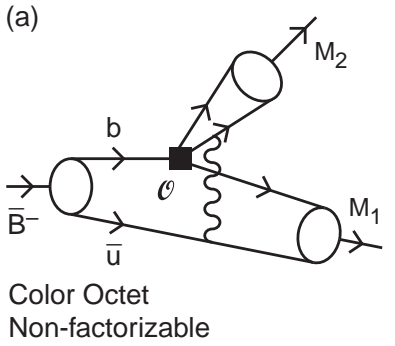

(b)

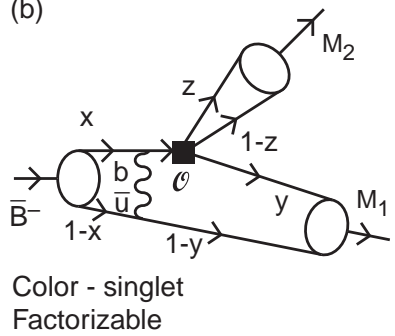

(c)

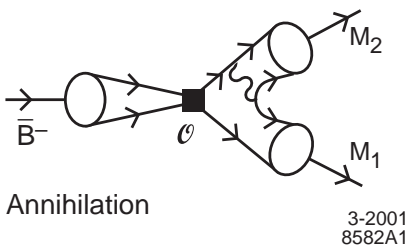

Figure 9: Three representative contributions to exclusive $B$ decays to meson pairs in PQCD. The operators $\mathcal{O}$ represent the QCD-improved effective weak interaction.

Here $x=k^{+} / p_{H}^{+}=\left(k^{0}+k^{z}\right) /\left(p_{H}^{0}+p_{H}^{z}\right)$ are the light-cone momentum fractions carried by the valence quarks.

There are a several features of QCD which are required to ensure the consistency of the PQCD approach: (a) the effective QCD coupling $\alpha_{s}\left(Q^{2}\right)$ needs to be under control at the relevant scales of $B$ decay; (b) the distribution amplitudes of the hadrons need to satisfy convergence properties at the endpoints; and (c) one requires the coherent cancellation of the couplings of soft gluons to color-singlet states. This property, color transparency [82], is a fundamental coherence property of gauge theory and leads to diminished final-state interactions and corrections to the PQCD factorizable contributions. The problem of setting the renormalization scale of the coupling for exclusive amplitudes is discussed in [62]. Conformal symmetry can be used as a guide to organize the hard scattering calculation and to determine the leading contributions to the hadron distribution amplitudes [83, 84, 85].

Baryon pair production in two-photon annihilation is also an important testing ground for QCD. The calculation of $T_{H}$ for Compton scattering requires the evaluation of 368 helicity-conserving tree diagrams which contribute to $\gamma(q q q) \rightarrow \gamma^{\prime}(q q q)^{\prime}$ at the Born level and a careful integration over singular intermediate energy denominators [86, 87, 88]. Brooks and Dixon [89 have completed a recalculation of the proton Compton process at leading order in PQCD, extending and correcting earlier work. It is useful to consider the ratio $d \sigma / d t(\gamma \gamma \rightarrow \bar{p} p) / d \sigma / d t\left(e^{+} e^{-} \rightarrow \bar{p} p\right)$ since the power-law fall-off, the normalization of the valence wavefunctions, and much of the uncertainty from the scale of the QCD coupling cancel. The scaling and angular dependence of this ratio is sensitive to the shape of the proton distribution amplitudes. The perturbative QCD predictions for the phase of the Compton amplitude phase can be tested in virtual Compton scattering by interference with Bethe-Heitler processes 90.

It is also interesting to measure baryon and isobar pair production in two photon reactions near threshold. Ratios such as $\sigma\left(\gamma \gamma \rightarrow \Delta^{++} \Delta^{--}\right) / \sigma\left(\gamma \gamma \rightarrow \Delta^{+} \Delta^{-}\right)$can be as large as $16: 1$ in the quark model since the three-quark wavefunction of the $\Delta$ is expected to be symmetric. Such large ratios would not be expected in soliton models 
[57] in which intermediate multi-pion channels play a major role.

Pobylitsa et al. [91] have shown how the predictions of perturbative QCD can be extended to processes such as $\gamma \gamma \rightarrow p \bar{p} \pi$ where the pion is produced at low velocities relative to that of the $p$ or $\bar{p}$ by utilizing soft pion theorems in analogy to soft photon theorems in QED. The distribution amplitude of the $p \pi$ composite is obtained from the proton distribution amplitude from a chiral rotation. A test of this procedure in inelastic electron scattering at large momentum transfer $e p \rightarrow p \pi$ and small invariant $p^{\prime} \pi$ mass has been remarkably successful. Many tests of the soft meson procedure are possible in multiparticle $e^{+} e^{-}$and $\gamma \gamma$ final states.

The leading-twist QCD predictions for exclusive two-photon processes such as the photon-to-pion transition form factor and $\gamma \gamma \rightarrow$ hadron pairs are based on rigorous factorization theorems. The data from the CLEO collaboration on $F_{\gamma \pi}\left(Q^{2}\right)$ and the sum of $\gamma \gamma \rightarrow \pi^{+} \pi^{-}$and $\gamma \gamma \rightarrow K^{+} K^{-}$channels are in excellent agreement with the QCD predictions. It is particularly compelling to see a transition in angular dependence between the low energy chiral and PQCD regimes. The success of leadingtwist perturbative QCD scaling for exclusive processes at presently experimentally accessible momentum transfer can be understood if the effective coupling $\alpha_{V}\left(Q^{*}\right)$ is approximately constant at the relatively small scales $Q^{*}$ relevant to the hard scattering amplitudes [62]. The evolution of the quark distribution amplitudes in the low- $Q^{*}$ domain also needs to be minimal. Sudakov suppression of the endpoint contributions is also strengthened if the coupling is frozen because of the exponentiation of a double logarithmic series.

One of the formidable challenges in QCD is the calculation of non-perturbative wavefunctions of hadrons from first principles. The calculations of the pion distribution amplitude by Dalley [64] and by Burkardt and Seal [92] using light-cone and transverse lattice methods is particularly encouraging. The predicted form of $\phi_{\pi}(x, Q)$ is somewhat broader than but not inconsistent with the asymptotic form favored by the measured normalization of $Q^{2} F_{\gamma \pi^{0}}\left(Q^{2}\right)$ and the pion wavefunction inferred from diffractive di-jet production.

Clearly much more experimental input on hadron wavefunctions is needed, particularly from measurements of two-photon exclusive reactions into meson and baryon pairs at the high luminosity $B$ factories. For example, as shown in Fig. 7 , the ratio

$$
\frac{d \sigma}{d t}\left(\gamma \gamma \rightarrow \pi^{0} \pi^{0}\right) / \frac{d \sigma}{d t}\left(\gamma \gamma \rightarrow \pi^{+} \pi^{-}\right)
$$

is particularly sensitive to the shape of pion distribution amplitude. At fixed pair mass, and high photon virtuality, one can study the distribution amplitude of multihadron states [55]. Two-photon annihilation will provide much information on fundamental QCD processes such as deeply virtual Compton scattering and large angle Compton scattering in the crossed channel. I have also emphasized the interrelation between the wavefunctions measured in two-photon collisions and the wavefunctions needed to study exclusive $B$ and $D$ decays. 
Much of the most interesting two-photon annihilation physics is accessible at low energy, high luminosity $e^{+} e^{-}$colliders, including measurements of channels important in the light-by-light contribution to the muon $g-2$ and the study of the transition between threshold production controlled by low-energy effective chiral theories and the domain where leading-twist perturbative QCD becomes applicable.

The threshold regime of hadron production in photon-photon and $e^{+} e^{-}$annihilation, where hadrons are formed at small relative velocity, is particularly interesting as a test of low energy theorems, soliton models, and new types of resonance production. Such studies will be particularly valuable in double-tagged reactions where polarization correlations, as well as the photon virtuality dependence, can be studied.

\section{New Calculational Methods}

The light-front quantization of gauge theories can be carried out in an elegant way using the Dirac method to impose the light-cone gauge constraint and eliminate dependent degrees of freedom [93]. Unlike the case in equal-time quantization, the vacuum remains trivial. Since only physical degrees of freedom appear, unitarity is maintained. One can verify the QCD Ward identities for the physical light-cone gauge and compute the QCD $\beta$ function. Recently, Srivastava and I [94 have extended the light-front quantization procedure to the Standard Model. The spontaneous symmetry breaking of the gauge symmetry is due to a zero mode of the scalar field rather than vacuum breaking. The Goldstone component of the scalar field provides mass to the $W^{ \pm}$and $Z^{0}$ gauge bosons as well as completing its longitudinal polarization. The resulting theory is free of Faddeev-Popov ghosts and is unitary and renormalizable. The resulting rules give an elegant new way to compute Standard model processes using light-front Hamiltonian theory.

The light-front method thus suggests the possibility of developing an "event amplitude generator" for high energy processes such as photon-photon collisions by calculating amplitudes for specific parton spins using light-front time-ordered perturbation theory 95. The positivity of the $k^{+}$light-front momenta greatly constrains the number of contributing light-front time orderings. The renormalized amplitude can be obtained diagram by diagram by using the "alternating denominator" method which automatically subtracts the relevant counterterm. The DLCQ method also provides a simple way to discretized the light-front momentum variables, while maintaining frame-independence. The resulting renormalized amplitude can be convoluted with the light-front wavefunctions to simulate hadronization and hadron matrix elements.

\section{Acknowledgments}

I thank Professor Clem Heusch for organizing and inviting me to this workshop and to David Asner, Jack Gunion, Dae Sung Hwang, Tom Rizzo, Ivan Schmidt, and Peter 
Zerwas for helpful comments. Work supported by the Department of Energy under contract number DE-AC03-76SF00515.

\section{References}

[1] For a review see, S. J. Brodsky and P. M. Zerwas, Nucl. Instrum. Meth. A 355, 19 (1995) arXiv:hep-ph/9407362.

[2] I. F. Ginzburg, G. L. Kotkin, V. G. Serbo and V. I. Telnov, JETP Lett. 34, 491 (1981) [Pisma Zh. Eksp. Teor. Fiz. 34, 514 (1981)].

[3] V. Telnov, 17th International Conference on High-Energy Accelerators (HEACC 98), Dubna, Russia, 7-12 Sep 1998 arXiv:hep-ex/9810019.

[4] D. Asner, S. Boege, J. Early, J. Gronberg, K. Skulina, K. van Bibber and T. Markiewicz, PAC-2001-FPAH055 Presented at IEEE Particle Accelerator Conference (PAC2001), Chicago, Illinois, 18-22 Jun 2001.

[5] M. M. Velasco et al., in Proc. of the APS/DPF/DPB Summer Study on the Future of Particle Physics (Snowmass 2001) ed. R. Davidson and C. Quigg, arXiv:hep-ex/0111055.

[6] See, e.g., D. M. Asner, J. B. Gronberg and J. F. Gunion, arXiv:hep-ph/0110320.

[7] B. Grzadkowski and J. F. Gunion, Phys. Lett. B 294, 361 (1992) arXiv:hepph/9206262].

[8] K. Cheung, arXiv:hep-ph/9310340.

[9] Wai-Keung Tang, unpublished.

[10] E. Boos et al., Nucl. Instrum. Meth. A 472, 100 (2001) arXiv:hep-ph/0103090.

[11] D. S. Hwang and S. J. Brodsky (unpublished).

[12] D. Asner et al., arXiv:hep-ex/0111056.

[13] S. J. Brodsky, SLAC-PUB-6314 Presented at the 2nd International Workshop on Physics and Experiments with Linear e+ e-Colliders, Waikoloa, HI, 26-30 Apr 1993.

[14] M. S. Chanowitz, Nucl. Instrum. Meth. A 355, 42 (1995) arXiv:hepph/9407231].

[15] J. F. Gunion and H. E. Haber, Phys. Rev. D 48, 5109 (1993).

[16] S. D. Drell and A. C. Hearn, Phys. Rev. Lett. 16, 908 (1966). 
[17] S. B. Gerasimov, Sov. J. Nucl. Phys. 2, 430 (1966) [Yad. Fiz. 2, 598 (1966)].

[18] S. J. Brodsky and J. R. Hiller, Phys. Rev. D 46, 2141 (1992).

[19] W. K. Tung, Phys. Rev. 176, 2127 (1968).

[20] S. J. Brodsky and S. D. Drell, Phys. Rev. D 22, 2236 (1980).

[21] E. Yehudai, Phys. Rev. D 44, 3434 (1991).

[22] J. Layssac and F. M. Renard, Phys. Rev. D 64, 053018 (2001) arXiv:hep$\mathrm{ph} / 0104205$.

[23] G. Jikia and A. Tkabladze, Phys. Lett. B 332, 441 (1994) arXiv:hep$\mathrm{ph} / 9312274$.

[24] G. J. Gounaris, P. I. Porfyriadis and F. M. Renard, Eur. Phys. J. C 20, 659 (2001) arXiv:hep-ph/0103135.

[25] G. V. Jikia, Nucl. Phys. B 374, 83 (1992).

[26] E. Boos, M. Dubinin, A. Pukhov, M. Sachwitz and H. J. Schreiber, Eur. Phys. J. C 21, 81 (2001) arXiv:hep-ph/0104279.

[27] S. J. Brodsky, T. G. Rizzo and I. Schmidt, Phys. Rev. D 52, 4929 (1995) arXiv:hep-ph/9505441.

[28] T. G. Rizzo, arXiv:hep-ph/9907395.

[29] I. F. Ginzburg, G. L. Kotkin, S. L. Panfil and V. G. Serbo, Nucl. Phys. B228, 285 (1983). See also S. Y. Choi and F. Schrempp, Phys. Lett. B272, 149 (1991); M. Raidal, Nucl. Phys. B441, 49 (1995), arXiv:hep-ph/9411243 .

[30] S. J. Brodsky, T. A. DeGrand, J. F. Gunion and J. H. Weis, Phys. Rev. D 19, 1418 (1979).

[31] M. Drees and T. Han, Phys. Rev. Lett. 76, 3076 (1996) arXiv:hep-ph/9512361.

[32] S. J. Brodsky, J. Rathsman and C. Merino, Phys. Lett. B 461, 114 (1999) arXiv:hep-ph/9904280.

[33] S. J. Brodsky, F. Hautmann and D. E. Soper, Phys. Rev. D 56, 6957 (1997) arXiv:hep-ph/9706427.

[34] J. Bartels, A. De Roeck and H. Lotter, Phys. Lett. B 389, 742 (1996) arXiv:hepph/9608401. 
[35] A. V. Lipatov and N. P. Zotov, Liverpool 2000, Deep Inelastic Scattering, pp. $157-158$

[36] V. S. Fadin and L. N. Lipatov, Phys. Lett. B 429, 127 (1998) arXiv:hep$\mathrm{ph} / 9802290]$.

[37] S. J. Brodsky, V. S. Fadin, V. T. Kim, L. N. Lipatov and G. B. Pivovarov, JETP Lett. 70, 155 (1999) [arXiv:hep-ph/9901229].

[38] S. J. Brodsky, T. Kinoshita and H. Terazawa, Phys. Rev. Lett. 27, 280 (1971).

[39] T. F. Walsh, Phys. Lett. B 36, 121 (1971).

[40] M. Krawczyk, Ambleside 2000, Structure and Interactions of the Photon arXiv:hep-ph/0012179.

[41] E. Witten, Nucl. Phys. B 120, 189 (1977).

[42] C. Peterson, P. M. Zerwas and T. F. Walsh, Nucl. Phys. B 229, 301 (1983).

[43] S. J. Brodsky, P. Hoyer, N. Marchal, S. Peigne and F. Sannino, arXiv:hep$\mathrm{ph} / 0104291$.

[44] S. J. Brodsky, D. S. Hwang and I. Schmidt, Phys. Lett. B 530, 99 (2002) arXiv:hep-ph/0201296.

[45] V. L. Chernyak and I. R. Zhitnitsky, Nucl. Phys. B 222, 382 (1983).

[46] I. F. Ginzburg, D. Y. Ivanov and V. G. Serbo, Phys. Atom. Nucl. 56, 1474 (1993) [Yad. Fiz. 56N11, 45 (1993)].

[47] D. Ashery, [E791 Collaboration], hep-ex/9910024.

[48] S. J. Brodsky and G. P. Lepage, Phys. Rev. Lett. 53, 545 (1979); Phys. Lett. 87B, 359 (1979); G. P. Lepage and S. J. Brodsky, Phys. Rev. D22, 2157 (1980).

[49] D. Asner and S. J. Brodsky, unpublished.

[50] M. R. Pennington, Ambleside 2000, Structure and Interactions of the Photon 388-393, arXiv:hep-ph/0009267.

[51] M. Acciarri et al. [L3 Collaboration], Phys. Lett. B 501, 173 (2001) hepex/0011037].

[52] A. Heister et al. [ALEPH Collaboration], Phys. Lett. B 530, 56 (2002) arXiv:hep-ex/0202011.

[53] S. J. Brodsky and G. P. Lepage, Phys. Rev. D24, 1808 (1981). 
[54] D. Muller, D. Robaschik, B. Geyer, F. M. Dittes, and J. Horejsi, Fortsch. Phys. 42, 101 (1994), hep-ph/9812448.

[55] M. Diehl, T. Gousset and B. Pire, Phys. Rev. D 62, 073014 (2000) hepph/0003233]. M. Diehl, T. Gousset, B. Pire and O. Teryaev, Phys. Rev. Lett. 81, 1782 (1998) arXiv:hep-ph/9805380.

[56] S. J. Brodsky, T. Kinoshita and H. Terazawa, Phys. Rev. D 4, 1532 (1971).

[57] H. Sommermann, M. R. Seki, S. Larson. and S. E. Koonin, Phys. Rev. D45, 4303 (1992); S. J. Brodsky and M. Karliner,(unpublished).

[58] M. Karliner and S. Nussinov, arXiv:hep-ph/0202234.

[59] S. J. Brodsky and C. R. Ji, Phys. Rev. Lett. 55, 2257 (1985).

[60] D. S. Hwang, these proceedings.

[61] S. J. Brodsky, M. Diehl and D. S. Hwang, Nucl. Phys. B 596, 99 (2001) arXiv:hep-ph/0009254.

[62] S. J. Brodsky, C. R. Ji, A. Pang and D. G. Robertson, Phys. Rev. D 57, 245 (1998) arXiv:hep-ph/9705221.

[63] J. Gronberg et al. [CLEO Collaboration], Phys. Rev. D57, 33 (1998), hepex/9707031].

[64] S. Dalley, Nucl. Phys. Proc. Suppl. 90, 227 (2000) arXiv:hep-ph/0007081.

[65] E. M. Aitala et al. [E791 Collaboration], Phys. Rev. Lett. 86, 4768 (2001) arXiv:hep-ex/0010043.

[66] A. V. Radyushkin, Acta Phys. Polon. B26, 2067 (1995).

[67] S. Ong, Phys. Rev. D52, 3111 (1995).

[68] P. Kroll and M. Raulfs, Phys. Lett. 387B, 848 (1996).

[69] V. L. Chernyak and A. R. Zhitnitsky, Phys. Rep. 112, 173 (1984).

[70] A. Khodjamirian, Eur. Phys. J. C 6, 477 (1999) arXiv:hep-ph/9712451.

[71] A. Schmedding and O. Yakovlev, Phys. Rev. D 62, 116002 (2000) arXiv:hepph/9905392].

[72] A. P. Bakulev, S. V. Mikhailov and N. G. Stefanis, Phys. Lett. B 508, 279 (2001) arXiv:hep-ph/0103119.

[73] A. Szczepaniak, E. M. Henley, and S. J. Brodsky, Phys. Lett. B243, 287 (1990). 
[74] A. Szczepaniak, Phys. Rev. D54, 1167 (1996).

[75] M. Beneke, G. Buchalla, M. Neubert and C. T. Sachrajda, Phys. Rev. Lett. 83, 1914 (1999) arXiv:hep-ph/9905312.

[76] Y. Y. Keum, H. n. Li and A. I. Sanda, Phys. Lett. B 504, 6 (2001) arXiv:hepph/0004004.

[77] Y. Y. Keum, H. N. Li and A. I. Sanda, Phys. Rev. D 63, 054008 (2001) arXiv:hep-ph/0004173.

[78] S. J. Brodsky and G. P. Lepage, Phys. Rev. D24, 2848 (1981).

[79] J. Dominick et al. [CLEO Collaboration], Phys. Rev. D 50, 3027 (1994) hepph/9403379]; J. Boyer et al., Phys. Rev. Lett. 56, 207 (1980); TPC/Two Gamma Collaboration (H. Aihara et al), Phys. Rev. Lett. 57, 404 (1986).

[80] M. Diehl, P. Kroll and C. Vogt, arXiv:hep-ph/0112274.

[81] S. J. Brodsky, Published in Ise-Shima 2001, B physics and CP violation, pp. 229-234. [hep-ph/0104153].

[82] S. J. Brodsky and A. H. Mueller, Phys. Lett. B206, 685 (1988).

[83] S. J. Brodsky, Y. Frishman, G. P. Lepage, and C. Sachrajda, Phys. Lett. 91B, 239 (1980).

[84] S. J. Brodsky, E. Gardi, G. Grunberg, and J. Rathsman, Phys. Rev. D63, 094017 (2001) [hep-ph/0002065].

[85] V. M. Braun, S. E. Derkachov, G. P. Korchemsky, and A. N. Manashov, Nucl. Phys. B553, 355 (1999) arXiv:hep-ph/9902375.

[86] G. R. Farrar and H. Zhang, Phys. Rev. Lett. 65, 1721 (1990).

[87] A. S. Kronfeld and B. Nizic, Phys. Rev. D44, 3445 (1991).

[88] P. A. Guichon and M. Vanderhaeghen, Prog. Part. Nucl. Phys. 41, 125 (1998), hep-ph/9806305.

[89] T. C. Brooks and L. J. Dixon, Phys. Rev. D 62, 114021 (2000) arXiv:hep$\mathrm{ph} / 0004143$.

[90] S. J. Brodsky, F. E. Close, and J. F. Gunion, Phys. Rev. D6, 177 (1972).

[91] P. V. Pobylitsa, . V. Polyakov and M. Strikman, Phys. Rev. Lett. 87, 022001 (2001) arXiv:hep-ph/0101279. 
[92] M. Burkardt and S. K. Seal, Phys. Rev. D 65, 034501 (2002) arXiv:hep$\mathrm{ph} / 0102245$.

[93] P. P. Srivastava and S. J. Brodsky, Phys. Rev. D 64, 045006 (2001) arXiv:hep$\mathrm{ph} / 0011372$.

[94] P. P. Srivastava and S. J. Brodsky, [arXiv:hep-ph/0202141].

[95] S. J. Brodsky, arXiv:hep-th/0111241. 